\title{
Bevacizumab is an Efficient Therapeutic Approach with Low Side Effects in Patient-Derived Xenografts of Adenoid Cystic Carcinoma of the Lacrimal Gland
}

\author{
Jingxue Zhang ${ }^{1,2, *}$, Xuejing Yan ${ }^{1} *$, Rui $\mathrm{Liu}^{3}$, Shen $\mathrm{Wu}^{1,2}$, Qian Liu ${ }^{1,2}$, Jing $\mathrm{Li}^{3}$, Jianmin $\mathrm{Ma}^{3}$ \\ 'Beijing Institute of Ophthalmology, Beijing Tongren Eye Center, Beijing Tongren Hospital, Capital Medical University, Beijing Ophthalmology \& Visual \\ Sciences Key Laboratory, Beijing, 100730, People's Republic of China; ${ }^{2}$ Beijing Institute of Brain Disorders, Collaborative Innovation Center for Brain \\ Disorders, Capital Medical University, Beijing, 100069, People's Republic of China; ${ }^{3}$ Beijing Tongren Eye Center, Beijing Tongren Hospital, Capital \\ Medical University, Beijing Ophthalmology \& Visual Sciences Key Laboratory, Beijing, I00730, People’s Republic of China
}

*These authors contributed equally to this work

Correspondence: Jianmin Ma, Beijing Tongren Eye Center, Beijing Tongren Hospital, Capital Medical University, Beijing Ophthalmology \& Visual Sciences Key Laboratory, No. I Dongjiaominxiang Street, Dongcheng District, Beijing, 100730, People's Republic of China, Tel +8610-5826-9968, Fax +86/0-5826-9930, Email jmma@sina.com

Purpose: Adenoid cystic carcinoma (ACC) of the lacrimal gland (LGACC) is an aggressive malignant lacrimal gland tumor with a generally poor prognosis. Survival rates for LGACC are $56 \%$ at 5 years and $49 \%$ at 10 years. Recent studies have indicated that antivascular endothelial growth factor (VEGF) therapy can inhibit angiogenesis in ACC cells. This study was designed to explore the efficacy of the antiangiogenic drug bevacizumab in a LGACC patient-derived xenograft (PDX) animal model.

Methods: The histological structure of PDX was determined by hematoxylin-eosin staining to confirm successful xenografting. Immunohistochemistry (IHC) was used to detect the expression of neovascularization-related genes in LGACC patients and in the PDX model, including VEGF, VEGFR1, and FGFR. In order to compare the efficacy of antiangiogenic drug and traditional chemotherapy drug, PDX models were treated with bevacizumab and cisplatin respectively, and body weight was evaluated. Subsequently, the neovascularization-related proteins VEGF, VEGFR2, and CD34, tumor suppressor P53 and proliferation-related protein Ki67 were analyzed by IHC. Quantitative real-time PCR was employed to examine the mRNA expression of apoptosis-related genes BAD and Caspase 9, and of HIF1 $\alpha$.

Results: VEGF, VEGFR1, and FGFR were highly expressed in patients with LGACC and PDX models. Both bevacizumab and cisplatin treatment inhibited PDX tumor growth. The body weight of PDX models treated with cisplatin significantly decreased from day 15, while those treated with bevacizumab did not markedly change. Bevacizumab reduced the expression of VEGF, CD34, and Ki67 in PDX tumors; whereas, bevacizumab upregulated P53 and downregulated HIF1 $\alpha$ levels.

Conclusion: The present study indicates that antiangiogenic drugs may be a promising treatment strategy for LGACC.

Keywords: LGACC, VEGF, PDX, bevacizumab, cisplatin

\section{Introduction}

Adenoid cystic carcinoma (ACC) is the most common malignant epithelial neoplasm of lacrimal glands, ${ }^{1}$ accounting for $25 \%$ to $40 \%$ of lacrimal epithelial neoplasms and for $1.6 \%$ of all orbital tumors. ${ }^{2,3}$ ACC is characterized by high recurrence rate of nearly $59 \%$, and early metastasis with approximately $20 \%$ to $50 \%$ of cases resulting in distant metastasis and poor prognosis. ${ }^{4-7}$ Currently, the treatment for ACC is surgical resection combined with radiation therapy, but more than $50 \%$ of ACCs recur or develop distant metastases over time. ${ }^{8}$ According to a previous study, LGACC can be treated locally with successful surgery and can be combined with radiation therapy even in advanced tumors, although 
5/7 patients still developed distant metastases 12 to 32 months after surgery. ${ }^{9}$ Consistently, Han et al demonstrated that, the rate of local cure for tumors of stages $\leq \mathrm{T} 3 \mathrm{a}$ is very high, also following eye-sparing surgery. ${ }^{10}$ Chemotherapy is another treatment option for ACC. However, the adverse effects of chemotherapy, including nausea, vomiting, myelosuppression, neutropenia, renal dysfunction, and hearing loss, limit the application of chemotherapy for ACC. Therefore, the identification of intervention drugs that can inhibit the proliferation of ACC of the lacrimal gland (LGACC) with low side effects has become a research hotspot.

Vascular endothelial growth factor (VEGF) is the key regulator of angiogenesis during tumor progression. Targeting VEGF signaling is commonly used to treat cancers, including glioma. ${ }^{11}$ Studies have shown that the knockdown of VEGF expression in ACC-2 cells-bearing nude mice could effectively inhibit tumor angiogenesis and tumor growth. ${ }^{12}$ Bevacizumab is a humanized monoclonal antibody against VEGF-A that directly binds VEGF-A, preventing activation of VEGF receptors. Bevacizumab has been approved for use in the treatment of multiple cancers, including colon cancer and ovarian cancer. ${ }^{13}$ However, the effectiveness of bevacizumab in ACC is largely unknown.

The patient-derived xenografts (PDX) model is a human xenotransplantation model established by direct transplantation of patient tumor tissue into immunodeficient mice. ${ }^{14}$ The PDX model is widely used in tumor drug screening and medication guidance. Drugs screened using PDX animal models have greater value for guiding clinical application of tumor treatment than do traditional animal tumor models. In this study, we evaluated the effectiveness of bevacizumab treatment in LGACC using a PDX animal model. Our results show that VEGF is highly expressed in patients with LGACC and that bevacizumab can effectively inhibit ACC PDX growth.

\section{Materials and Methods}

\section{Patient Samples}

Eight LGACC tissue samples and normal lacrimal gland tissue samples were obtained from the Beijing Tongren Hospital between November and December 2019. Three tissues were used to establish PDX, and others were used to detect VEGF signaling markers. The tissues used for the establishment of PDX models were from patients without any treatment. LGACC pathology was confirmed by two different specialist diagnoses. The involvement of human participants in this study was reviewed and approved by the Beijing Tongren Hospital and this study was performed in accordance with the Declaration of Helsinki. All patients/participants provided their written informed consent to participate in this study.

\section{Establishment of PDX Model and Drug Intervention}

The animal study was approved and monitored by the Institutional Animal Care and Use Committee of the Capital Medical University of Beijing (IACUC; AEEI-2018-198), and it conformed to the National Institute of Health Guide for the Care and Use of Laboratory Animals as well as the Association for Research in Vision and Ophthalmology (ARVO) Statement for the Use of Animals in Ophthalmic and Vision Research.

Tissues surgically removed from patients with LGACC were transported on ice to the specific pathogen-free animal room (all subsequent operations were completed on ice). Patient-derived LGACC tissues were cut into $20-30 \mathrm{~mm}^{3}$ pieces and inoculated into the back of NOD SCID mice to establish the PDX model. These mice were designated as passage 0 (P0). LGACC tissue derived from 1 patient inoculated 5 mice, and each mouse was inoculated with a single piece of tissue. Mice were euthanized when the tumor size reached $1500 \mathrm{~mm}^{3}$, and tumor tissues were removed and re-inoculated into new mice to obtain subsequent passages, namely, P1, P2, and P3. Using this approach, the PDX P3 model was established.

Drug intervention commenced once the tumor reached a volume of $100 \mathrm{~mm}^{3}$. Mice were randomly divided into the control group, bevacizumab (Roche, Basel, Switzerland, CAS No: 216974-75-3) intervention group, and cisplatin (Shanghai Yuanye Biotechnology, B24462) intervention group, with 7 mice in each group. The control group was intravenously injected with normal saline twice weekly, while the bevacizumab treatment group was intravenously injected with $5 \mathrm{mg} / \mathrm{kg}$ bevacizumab twice weekly. In the cisplatin treatment group, $2.5 \mathrm{mg} / \mathrm{kg}$ cisplatin was administered by tail vein injection once a week. Tumor sizes were measured every 3 days, and tumor volume was calculated using the following formula: $V=1 / 2 * a b^{2}$, where, $a$ and $b$ are the tumor length and width, respectively. 
The experiment lasted for 33 days, and afterwards the mice were euthanized. Tumors were separated and preserved in liquid nitrogen and formalin.

\section{Hematoxylin and Eosin Staining of Tumor Specimens in PDX Model}

Tumor specimens were dewaxed and rehydrated using the same procedure used for immunohistochemistry describe below. Specimens were impregnated with Harris hematoxylin for 6 minutes, washed with water for 2 minutes, and differentiated with $1 \%$ hydrochloric acid ethanol for 5 seconds. After washing for 7 minutes and dyeing for 2 minutes, the specimens were quickly rinsed with water, dehydrated, sealed, and fixed. Then, pictures were taken under an optical microscope.

\section{Immunohistochemical Staining}

Fixed tissues, including clinical samples of LGACC, PDX model tumor specimens, and PDX model tumor specimens after drug intervention, were dehydrated, paraffin impregnated, embedded, and sliced using immunohistochemical streptavidin-peroxidase methods. Dehydration was performed using xylene and $95 \%$ ethanol in turn, and samples were finally soaked in steamed water twice for 5 minutes each. After microwave antigen repair of citrate, slices were rinsed with phosphate buffered saline (PBS) twice for 3 minutes, and treated with $3 \%$ hydrogen peroxide $\left(37^{\circ} \mathrm{C}\right)$ for 10 minutes to block endogenous peroxidase activity. Samples were then blocked with serum for 25 minutes at $37^{\circ} \mathrm{C}$ and directly incubated with primary antibody at $4^{\circ} \mathrm{C}$ overnight. The next day, after washing with PBS, samples were incubated with the secondary antibody at $37^{\circ} \mathrm{C}$ for 25 minutes. The samples were then washed with PBS and incubated with Streptomyces coupled with horseradish peroxidase-labeled streptomycin ovalbumin for 15 minutes at $37^{\circ} \mathrm{C}$. Samples were then washed with PBS and stained with diaminobenzidine. After washing with distilled water, the slices were sealed with neutral resin, observed, and photographed under a light microscope. The positivity was calculated by the ratio of positive cells/total cells, which was analyzed using Image J software. The anti-VEGF (Cat No. 19003-1-AP), antiVEGFR1 (Cat No. 13687-1-AP), anti-VEGFR2 (Cat No. 26415-1-AP), anti-FGFR1 (Cat No. 60325-1-IG), anti-CD34 (Cat No. 14486-1-AP), and anti-Ki67 (Cat No. 27309-1-AP) primary antibodies were purchased from Proteintech. The anti-PDGF (Cat No. 3174) was purchased from CST. All primary antibodies were used at a 1:50 dilution. HRPconjugated anti-mouse (G1214) or anti-rabbit secondary antibodies (G1231) (dilution rate, 1:50) were purchased from Servicebio (Wuhan, China).

\section{Quantitative Real-Time Polymerase Chain Reaction (qRT-PCR)}

Total RNA was extracted from the tumor tissue samples obtained from the PDX model using Trizol (Invitrogen, Cat No.15596018). The RNA was reversely transcribed into cDNA using M-MLV reverse transcriptase (Promega, M1701). Quantification of specific cDNA sequences was performed on the qPCR platform by Biorad with the SYBR Master Mixture (TransGene, AQ311-01). The qPCR primer sequences used were as follows: Bad forward, 5'CCCAGAGTTTGAGCCGAGTG-3' and reverse, 5'-CCCATCCCTTCGTCGTCCT-3'; caspase-9 forward, 5'CTCAGACCAGAGATTCGCAAAC-3' and reverse, 5'-GCATTTCCCCTCAAACTCTCAA-3'; HIF1 $\alpha$ forward, 5'GAACGTCGAAAAGAAAAGTCTCG-3' and reverse, 5'-CCTTATCAAGATGCGAACTCACA-3'; $\beta$-actin forward, 5'-CATGTACGTTGCTATCCAGGC-3' and reverse, 5'-CTCCTTAATGTCACGCACGAT-3'.

\section{Statistical Analysis}

SPSS software version 22.0 (SPSS, Chicago, IL, USA) was used for processing. Data are described as mean \pm standard error (mean $\pm \mathrm{SD}$ ). Multivariate data were assessed using ANOVA, and pairwise comparison was performed using the Fisher's least significant difference test. $\mathrm{P}<0.05$ was considered statistically significant.

\section{Results}

\section{VEGF, VEGFRI, VEGFR2, and FGFR Were Highly Expressed in LGACC Samples}

We first detected the expression of VEGF, VEGFR1, VEGFR2, and FGFR in LGACC clinical samples and normal control tissues. The results showed that VEGF, VEGFR1, VEGFR2, and FGFR expression was significantly higher in 
LGACC tissues than in normal lacrimal gland tissues. In tumor tissues, the VEGF, VEGFR1, VEGFR2, and FGFR1 positivity rates were approximately $82.2 \%, 66.5 \%, 61.6 \%$, and $59.3 \%$, respectively (Figure 1).

\section{The Characteristics of LGACC Patients Whose Samples Were Used in PDX Model Construction}

We collected 5 LGACC samples for PDX model construction. The characteristics of patients with LGACC are listed in Table 1. Only 1 of 3 samples from LGACC patients developed tumors in the PDX mouse model. The tumors were subjected to hematoxylin and eosin staining (Figure 2A) and IHC staining. VEGF, VEGFR1, and VEGFR2 are highly expressed in PDX tumors derived from patients with LGACC (Figure 2B-E). These results indicate that the PDX model was successfully constructed in LGACC.

\section{Anti-Angiogenesis Drugs Significantly Inhibited the Proliferation of the LGACC PDX Model}

To explore the therapeutic effects of anti-neovascular drugs on the PDX model of LGACC, we divided the PDX model into three groups, namely the control group, the bevacizumab treatment group, and the cisplatin treatment group. Both bevacizumab and cisplatin treatment significantly inhibited the proliferation of PDX tumors (Figure 3A-C). The volumes of tumors in the intervention group were significantly lower than those in the control group at different time points (Figure $3 \mathrm{C}, \mathrm{p}<0.001$ ).
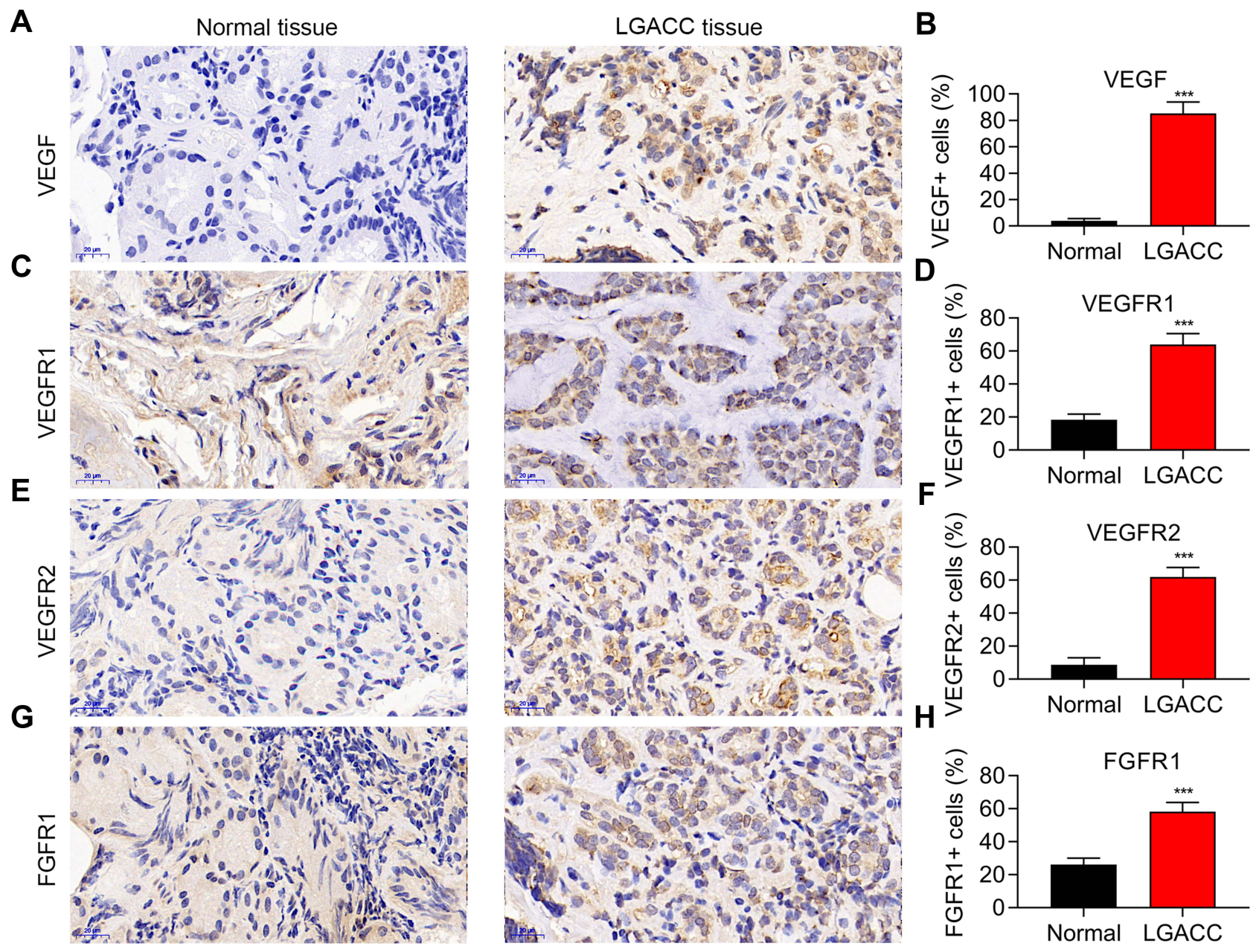

Figure I Neovascularization related proteins are highly expressed in LGACC tissues. VEGF (A), VEGFRI (C), VEGFR2 (E), and FGFRI (G) expression in LGACC and normal tissues detected by immunohistochemistry, and the related statistical analysis (B, D, $\mathbf{F}$ and $\mathbf{H})$. ${ }^{* * *} \mathrm{p}<0.00 \mathrm{I}$. $\mathrm{n}=5$. 
Table I Characteristics of Patients with LGACC

\begin{tabular}{|l|c|c|c|}
\hline & Patient I & Patient 2 & Patient 3 \\
\hline PDX establishment & Failure & Failure & Successful \\
\hline Gender & Female & Female & 73 \\
\hline Age & 39 & 34 & Tubular type \\
\hline Pathological type & High level transformation & Sieve type & None \\
\hline Metastasis & None & None & Yes \\
\hline Recurrence & None & Yes & ck(+), ck7(+), sox-10(+), ki-67(10\%), \\
\hline IHC characteristics & $c k(+), c k 7(+)$, sox-10(+), ki-67(30\%), & k63-67(2\%), p63(+) & \\
\hline
\end{tabular}

Tumor weight was significantly reduced in both the bevacizumab and cisplatin groups (Figure 3B). Compared with the control group, the body weight of mice in the cisplatin treatment group decreased significantly from the 15th day after the intervention, while the body weight of the mice in the bevacizumab treatment group underwent minimal changes (Figure 3D). Taken together, these data show that both bevacizumab and cisplatin significantly suppressed the growth of LGACC-derived PDX tumors. Compared with cisplatin, bevacizumab exhibited marginal side effects in the mice.

\section{Anti-Angiogenesis Drugs Suppressed Angiogenesis and Cell Proliferation in PDX Tumors}

To clarify the molecular changes following bevacizumab and cisplatin treatment, we performed IHC staining to assess the expression of proliferation and angiogenesis-related markers. In PDX tumor tissues treated with bevacizumab and cisplatin, the expression of Ki67, a positive regulator of proliferation, was reduced, while the expression of P53, a negative regulator of proliferation, was significantly upregulated (Figure 4A and C). The CD34 neovascularization biomarker was significantly downregulated in bevacizumab and cisplatin-treated tumor tissues (Figure 4B). Interestingly,

A

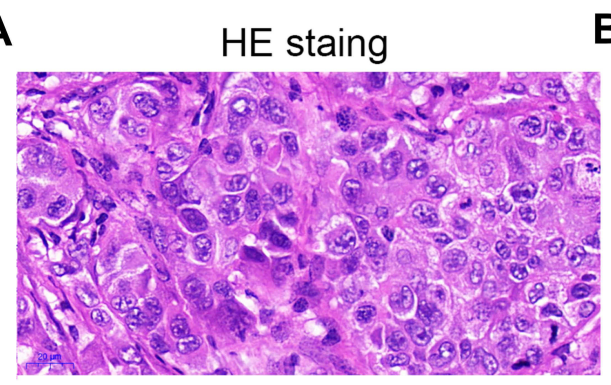

B VEGF

C

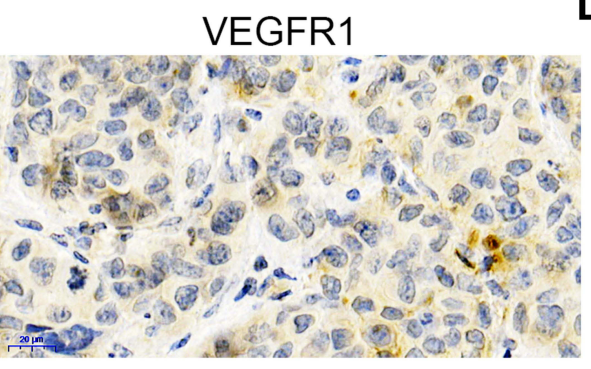

D
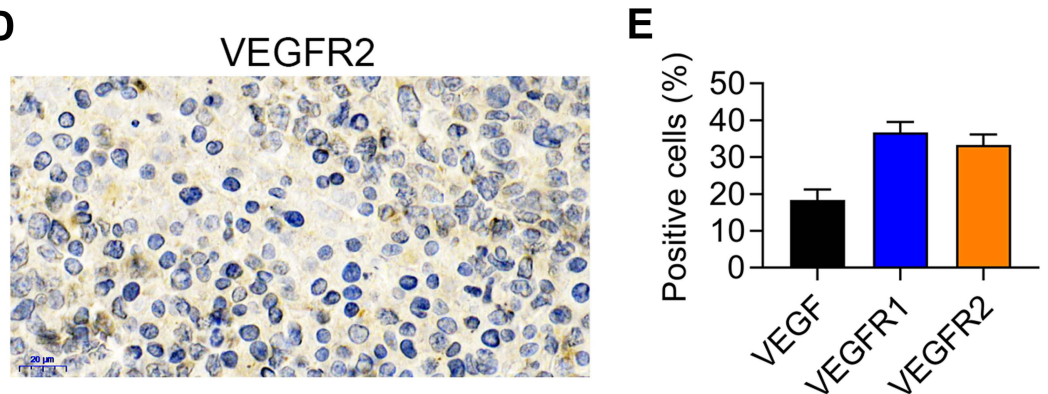

Figure 2 Patient-derived xenograft (PDX) model characterization. (A) Hematoxylin and Eosin staining of PDX tissue. (B-D) VEGF (B), VEGFRI (C), and VEGFR2 (D) expression in the PDX model detected by immunohistochemical staining. (E) The statistical analysis of (B-D). $n=5$. 
A

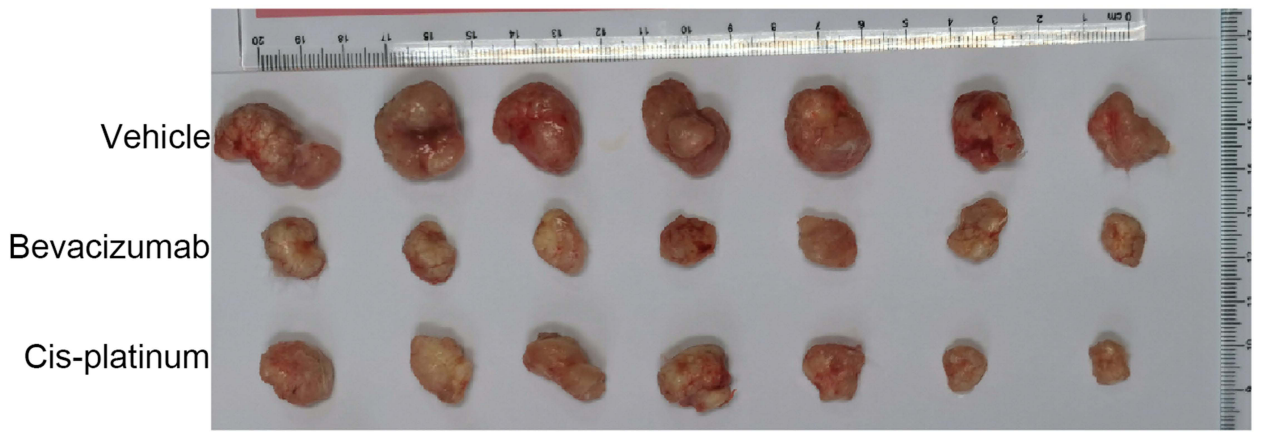

C

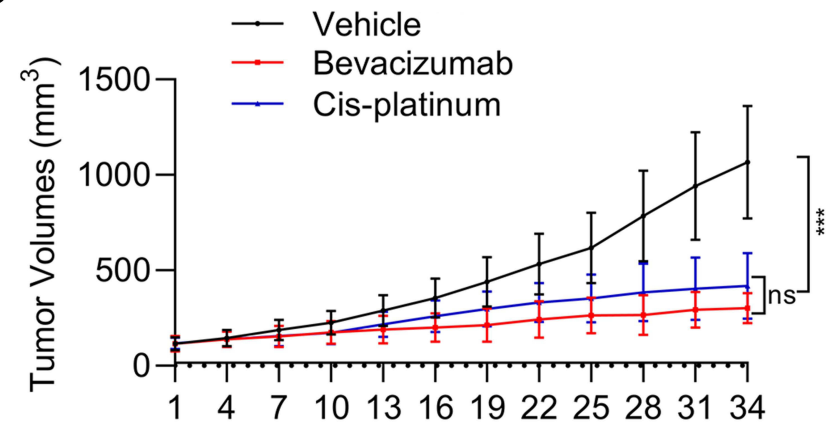

Day after Injection
B
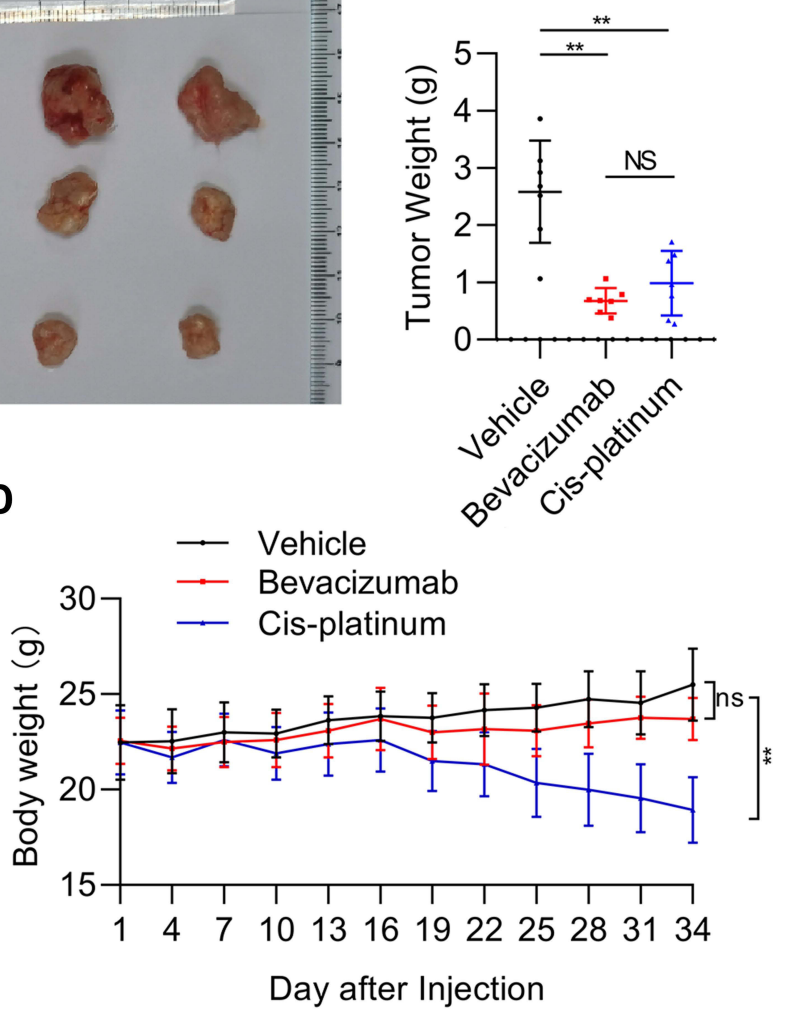

Figure 3 The efficacy of bevacizumab and cisplatin on patient-derived xenograft (PDX) tumor growth and body weight of mice. (A) Representative images of PDX tumor in vehicle, bevacizumab treatment, and cisplatin treatment groups. (B) PDX tumor weight in vehicle, bevacizumab treatment, and cisplatin treatment groups. ** $<0.0$ I; NS, not significant. (C) PDX tumor growth curves. (D) Body weight of PDX mice following vehicle, bevacizumab, and cisplatin treatment. ** $<<0.01$; *** $<<0.00 \mathrm{I}$. $\mathrm{n}=7$. NS, not significant.

the abundance of VEGF protein, but not VEGFR1 protein, was reduced in the bevacizumab-treatment group (Figure 4D and E). Cisplatin treatment had no effect on VEGF and VEGFR1 expression (Figure 4D and E). Moreover, the mRNA levels of Bad and Caspase 9 did not change and HIF1 $\alpha$ was downregulated in the bevacizumab-treatment group (Figure 5).

\section{Discussion}

In this study, we first identified higher levels of VEGF expression and its receptors, in LGACC samples compared with normal control tissues. Next, we successfully constructed a PDX animal model of LGACC and detected the antitumor effects of bevacizumab and cisplatin. Both bevacizumab and cisplatin effectively inhibited the growth of PDX. Importantly, while the body weight decreased during cisplatin treatment, there was minimal change in mouse body weight after bevacizumab treatment. Moreover, both bevacizumab and cisplatin treatment led to P53 upregulation and Ki-67 and CD34 downregulation. ${ }^{15}$ VEGF expression was downregulated in the bevacizumab treatment group only. Investigation of the molecular changes after bevacizumab and cisplatin treatment revealed the mRNA expression of proapoptotic genes Bad and caspase-9 were upregulated after cisplatin, but not after bevacizumab treatment. Moreover, expression of HIF $1 \alpha$ protein, a downstream factor of VEGF signaling, was downregulated in the bevacizumab treatment group but not in the cisplatin treatment group. These results indicate that bevacizumab treatment suppresses neovascularization to inhibit tumor cell proliferation.

Lack of an effective therapeutic strategy, inadequate initial treatment, as well as patients at advanced stage has contributed to the poor prognosis of patients with LGACC, that LGACC $>\mathrm{T} 3$ at initial diagnosis associated with worse 

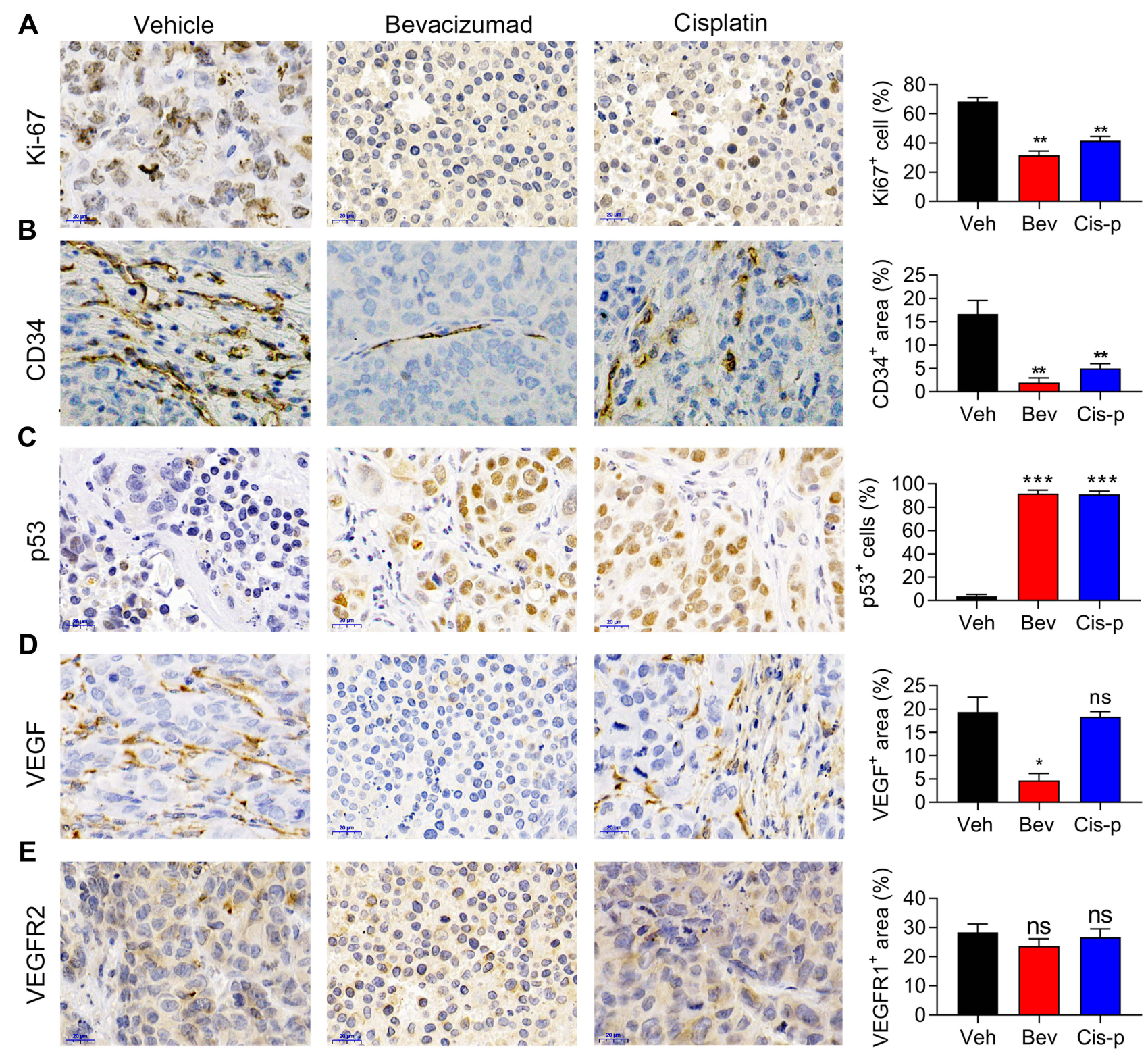

Figure 4 Bevacizumab and cisplatin suppress angiogenesis and cell proliferation in patient-derived xenograft (PDX) tumors. (A-E) Expression of Ki-67 (A), CD34 (B), P53 (C), VEGF (D), and VEGFR2 (E) detected by immunohistochemical staining in vehicle, bevacizumab treatment, and cisplatin treatment groups. *p < 0.01 ; **p < 0.05 ; *** $<$ 0.00I. $n=5$.

NS, not significant.

outcomes. ${ }^{4,16}$ Surgical resection combined with radiotherapy/chemotherapy, including ${ }^{125}$ I implanted internal radiotherapy, local external radiotherapy, or transarterial interventional chemotherapy, is the most common approach used to treat patients with LGACC. ${ }^{17}$ However, the clinical outcomes of these treatments are far from satisfactory. In recent years, targeted therapy has become a new strategy for cancer treatment, including targeted therapy for LGACC. Chen et al performed fluorescence in situ hybridization on 12 cases of LGACC using an MYB probe. Using this approach, the authors identified a MYB and NFIB fusion gene, indicating that this fusion gene might be a site for targeted therapy. ${ }^{18}$ Using exon sequencing, Sant et al found that the bromine region PHD domain transcription factor (BPTF), a Notch signaling pathway gene, was the most common mutated gene in low-grade ACC, indicating that the Notch pathway may be a potential target for the treatment of low-grade ACC. ${ }^{19}$ In addition, B cell lymphoma-2 (Bcl-2) overexpression and the activation of the NF- $\mathrm{KB}$ signaling pathway also promote ACC development. ${ }^{20}$ In this context, a clinical study of the effect of treatment with the NF- $\mathrm{KB}$ inhibitor bortezomib in 25 patients with advanced ACC of the head and neck revealed 

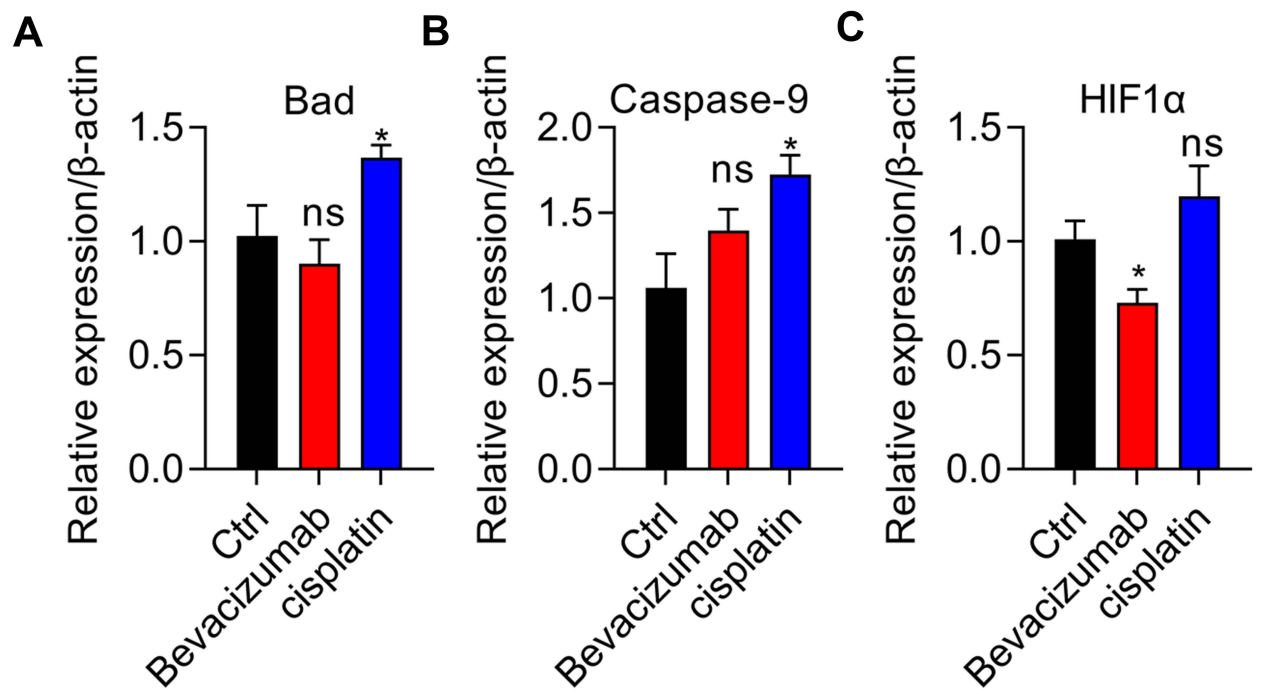

Figure 5 Cisplatin elevates apoptotic gene expression and bevacizumab suppresses HIFI $\alpha$ expression. (A and B) Proapoptotic-related mRNA expression of genes Bad (A) and Caspase-9 (B) was assessed using quantitative real-time PCR in vehicle, bevacizumab, and cisplatin treatment groups. *P < $0.0 \mathrm{I}$. (C) Examination of HIFI $\alpha$ expression using quantitative real-time PCR in vehicle, bevacizumab treatment, and cisplatin treatment groups. *p $<0.01$. $n=3$.

NS, not significant.

that the overall patient conditions are stable, but the clinical value in the application of this treatment requires further study. ${ }^{21}$

The translation of fundamental research to clinical application is the main challenge for targeted therapy. Moreover, lack of appropriate animal models for drug screening remains a major difficulty for these studies. Several studies have shown that survivin inhibits the growth of ACC-2 xenografted tumors via down-regulating VEGF expression, subsequently suppressing tumor angiogenesis and inducing tumor cell apoptosis. ${ }^{12,22}$ However, such animal tumor models were constructed by transplantation of tumor cell lines in nude mice. There are still limitations in the significance of such models for guiding clinical application. Recent studies involving xenotransplantation models of human tumors, named PDX models, provide a better research direction. ${ }^{14}$ The PDX model has been widely applied for drug screening in human cancers, including melanoma ${ }^{23}$ and pancreatic cancer. ${ }^{24}$ However, the tumor establishment rate is still dissatisfactory in some cancer types. For example, a previous study collected 168 solid tumors from pediatric patients and only successfully established 67 PDX models. ${ }^{25}$ In this study, we successfully constructed one PDX animal model derived from three human LGACC samples. This study provides the first evidence that PDX could be established from a patient with LGACC, although the sample numbers were very limited. Thus, we will collect more samples to establish PDX studies in the future. Based on this model, in the present study, we demonstrated that bevacizumab effectively suppresses LGACC tumor development, suggesting that targeting VEGF and angiogenesis might be a promising therapeutic strategy for LGACC.

Combinations of targeted therapy and chemotherapy have been used to treat malignant tumors, including ACC. Doddapaneni et al performed high-throughput proteomic analysis on six cases of LGACC and found that fibroblast growth factor (FGF) was upregulated, and the FGF receptor 1 (FGFR1) signaling pathway was significantly activated in ACC. They evaluated the therapeutic effects of a novel FGFR1-selective inhibitor, AZD4547, combined with cisplatin for the treatment of ACC. Their results showed that FGFR1 inhibition enhanced the cellular toxicity effect of cisplatin. ${ }^{26}$ Herein, we did not explore whether the application of combined anti-angiogenesis drugs can promote the inhibitory effect of platinum-based chemotherapy, and this needs further experimental confirmation. Of great significance for clinical applications is the investigation of the joint application of anti-neovascular drugs and platinum-based drugs in future research. A limitation of this study is that we did not perform genetic testing or molecular typing on the clinical samples. Whether LGACC of different genetic backgrounds responds differently to anti-angiogenic drugs still needs to be determined. 


\section{Conclusion}

In summary, we provide the first evidence that a PDX model can be established from using tissue samples from a patient with LGACC. This preclinical mouse model of LGACC can be used for drug screening of chemotherapies, targeted therapies, or even immunotherapies. In addition, we showed that bevacizumab performed better than cisplatin for LGACC treatment, as confirmed by higher inhibitory effects and lower side effects. Our results demonstrate that antiangiogenesis inhibitors are promising drugs for the clinical treatment of LGACC.

\section{Ethical Statement}

The patients/participants provided their written informed consent to participate in this study and this study was approved and monitored by the Institutional Animal Care and Use Committee of the Capital Medical University of Beijing (IACUC; AEEI-2018-198).

\section{Acknowledgments}

We thank post-doctoral student Ying Cheng for the English language editing.

\section{Funding}

This work was supported by the National Natural Science Foundation of China (grant \#81800864), Beijing Municipal Institute of Public Medical Research Development and Reform Pilot Project (grant \#2018-2), Beijing Hospitals Authority Ascent Plan (grant \#DFL20190201), and Beijing Hospitals Authority Youth Programme (grant \#QML20180208).

\section{Disclosure}

The authors declare that they have no conflicts of interest in this work.

\section{References}

1. Chawla B, Kashyap S, Sen S, et al. Clinicopathologic review of epithelial tumors of the lacrimal gland. Ophthal Plast Reconstr Surg. 2013;29 (6):440-445. doi:10.1097/IOP.0b013e31829f3a0c

2. Esmaeli B, Ahmadi MA, Youssef A, et al. Outcomes in patients with adenoid cystic carcinoma of the lacrimal gland. Ophthal Plast Reconstr Surg. 2004;20(1):22-26. doi:10.1097/01.IOP.0000105518.72611.4F

3. Yang J, Zhou C, Wang Y, Fan X, Jia R. Multimodal therapy in the management of lacrimal gland adenoid cystic carcinoma. BMC Ophthalmol. 2019;19(1):125. doi:10.1186/s12886-019-1110-5

4. Ahmad SM, Esmaeli B, Williams M, et al. American Joint Committee on Cancer classification predicts outcome of patients with lacrimal gland adenoid cystic carcinoma. Ophthalmology. 2009;116(6):1210-1215. doi:10.1016/j.ophtha.2008.12.049

5. Andreasen S, Esmaeli B, Holstein SL, Mikkelsen LH, Rasmussen PK, Heegaard S. An update on tumors of the lacrimal gland. Asia Pac J Ophthalmol. 2017;6(2):159-172. doi:10.22608/APO.201707

6. Lesueur P, Rapeaud E, De Marzi L, et al. Adenoid cystic carcinoma of the lacrimal gland: high dose adjuvant proton therapy to improve patients outcomes. Front Oncol. 2020;10:135. doi:10.3389/fonc.2020.00135

7. Armstrong LTD, Subramaniam SS, Borgna S. Adenoid cystic carcinoma of the palate with isolated cutaneous metastasis: a unique case and review of current and future treatment modalities of metastatic disease. Int J Oral Maxillofac Surg. 2020;49(6):700-708. doi:10.1016/j.ijom.2019.11.012

8. Ho AS, Ochoa A, Jayakumaran G, et al. Genetic hallmarks of recurrent/metastatic adenoid cystic carcinoma. J Clin Invest. 2019;129(10):42764289. doi:10.1172/JCI128227

9. Esmaeli B, Golio D, Kies M, DeMonte F. Surgical management of locally advanced adenoid cystic carcinoma of the lacrimal gland. Ophthalmic Plast Reconstr Surg. 2006;22(5):366-370. doi:10.1097/01.iop.0000232164.00208.b4

10. Han J, Kim YD, Woo KI, Sobti D. Long-term outcomes of eye-sparing surgery for adenoid cystic carcinoma of lacrimal gland. Ophthalmic Plast Reconstr Surg. 2018;34(1):74-78. doi:10.1097/IOP.0000000000000877

11. Nishikawa M, Inoue A, Ohnishi T, et al. CD44 expression in the tumor periphery predicts the responsiveness to bevacizumab in the treatment of recurrent glioblastoma. Cancer Med. 2021;10(6):2013-2025. doi:10.1002/cam4.3767

12. Fu K, Yang J, Wang X, Xu B. Survivin siRNA suppresses angiogenesis of adenoid cystic carcinoma ACC-2 cells-bearing nude mice. $J$ Third Mil Med Univ. 2010;32:4.

13. Haunschild CE, Tewari KS. Bevacizumab use in the frontline, maintenance and recurrent settings for ovarian cancer. Future Oncol. 2020;16 (7):225-246. doi:10.2217/fon-2019-0042

14. Stewart E, Federico SM, Chen X, et al. Orthotopic patient-derived xenografts of paediatric solid tumours. Nature. 2017;549(7670):96-100. doi:10.1038/nature23647

15. Bajpai M, Pardhe N. Immunohistochemical Expression of CD-117 (c-KIT), P-53 and Ki-67 in adenoid cystic carcinoma of palate. J Coll Physicians Surg Pak. 2018;28(6):S130-S132. doi:10.29271/jcpsp.2018.06.S130

16. Zhang M, Fathy C, Breazzano MP, Hollar M, Barahimi B. Intra-arterial chemotherapy for lacrimal gland adenoid cystic carcinoma. Int Ophthalmol Clin. 2017;57(1):143-152. doi:10.1097/IIO.0000000000000158 
17. Liu R, Shi JT, Ge X, et al. Similar therapeutic effects of 125 I seed radiotherapy and $\gamma$-ray radiotherapy on lacrimal gland adenoid cystic carcinoma. Int J Ophthalmol. 2021;14(4):547. doi:10.18240/ijo.2021.04.11

18. Chen TY, Keeney MG, Chintakuntlawar AV, et al. Adenoid cystic carcinoma of the lacrimal gland is frequently characterized by MYB rearrangement. Eye. 2017;31(5):720-725. doi:10.1038/eye.2016.307

19. Sant DW, Tao W, Field MG, et al. Whole exome sequencing of lacrimal gland adenoid cystic carcinoma. Invest Ophthalmol Vis Sci. 2017;58(6): BIO240-BIO246. doi:10.1167/iovs.16-21097

20. Wang XF, Wu DM, Li BX, Lu YJ, Yang BF. Synergistic inhibitory effect of sulforaphane and 5-fluorouracil in high and low metastasis cell lines of salivary gland adenoid cystic carcinoma. Phytother Res. 2009;23(3):303-307. doi:10.1002/ptr.2618

21. Argiris A, Ghebremichael M, Burtness B, Axelrod RS, Deconti RC, Forastiere AA. A Phase 2 trial of bortezomib followed by the addition of doxorubicin at progression in patients with recurrent or metastatic adenoid cystic carcinoma of the head and neck: a trial of the Eastern Cooperative Oncology Group (E1303). Cancer. 2011;117(15):3374-3382. doi:10.1002/cncr.25852

22. Wang X, Yang J, Xu B, et al. Effects of survivin gene silencing on growth and apoptosis of adenoid cystic carcinoma cell line. $J$ Third Mil Med Univ. 2009;31(11):4.

23. Cancer Genome Atlas Research Network. Integrated genomic analyses of ovarian carcinoma. Nature. 2011;474(7353):609-615. doi:10.1038/ nature 10166

24. Garcia P, Miller A, Yoon K. Patient-derived xenograft models of pancreatic cancer: overview and comparison with other types of models. Cancers. 2020;12(5):1327. doi:10.3390/cancers 12051327

25. Stewart E, Federico S, Chen X, et al. Orthotopic patient-derived xenografts of paediatric solid tumours. Nature. 2017;549(7670):96-100.

26. Doddapaneni R, Tao W, Naranjo A, Nikpoor N, Tse DT, Pelaez D. Fibroblast growth factor receptor 1 (FGFR1) as a therapeutic target in adenoid cystic carcinoma of the lacrimal gland. Oncotarget. 2019;10(4):480-493. doi:10.18632/oncotarget.26558

\section{Publish your work in this journal}

Cancer Management and Research is an international, peer-reviewed open access journal focusing on cancer research and the optimal use of preventative and integrated treatment interventions to achieve improved outcomes, enhanced survival and quality of life for the cancer patient. The manuscript management system is completely online and includes a very quick and fair peer-review system, which is all easy to use. Visit http://www.dovepress.com/testimonials.php to read real quotes from published authors.

Submit your manuscript here: https://www.dovepress.com/cancer-management-and-research-journal 